\title{
A função reflexiva em adolescentes em conflito com a lei e em adolescentes escolares
}

\author{
Taís Cristina Favaretto ${ }^{1}$ \\ Luciane Maria Both (1) $^{2}$ \\ Sílvia Pereira da Cruz Benetti@ 1 \\ ${ }^{1}$ Universidade do Vale do Rio dos Sinos, RS, Brasil. \\ ${ }^{2}$ Universidade Federal do Rio Grande do Sul, RS, Brasil.
}

\begin{abstract}
Resumo
A função reflexiva (FR) é identificada como um constructo importante à compreensão psicopatológica e para a prática clínica. Sendo assim, neste artigo, busca-se ampliar o conhecimento sobre a função reflexiva em dois grupos de adolescentes com diferentes trajetórias, um em conflito com a lei $(\mathrm{G} 1=91$, $\mathrm{M}$ idade 16,57 anos, $\mathrm{DP}=0,77)$ e outro de escolares $(\mathrm{G} 2=64$, $\mathrm{M}$ de idade 15,89 anos, $\mathrm{DP}=0,75)$. Os instrumentos utilizados foram dados sociodemográficos, Questionário sobre Função Reflexiva para Jovens e Questionário de Capacidade e Dificuldades. Da amostra, identificou-se que 119 adolescentes eram não clínicos e 95 clínicos. A análise univariada demonstrou diferenças significativas na função reflexiva considerando a interação entre a variável G1 e G2 e as categorias clínico e não clínico. Assim, o G2 não clínico apresentou maior função reflexiva $(\mathrm{M}=8,60 ; \mathrm{DP}=0,68 ; \mathrm{F}=4,66 ; \mathrm{p}<0,05)$, seguido pelo $\mathrm{G} 1$ clínico $(\mathrm{M}=8,29 ; \mathrm{DP}=0,68)$. Tal característica no grupo clínico de adolescentes em conflito com a lei assinala hipermentalização. Aponta-se, portanto, a necessidade de avaliação e desenvolvimento da função reflexiva como prevenção em saúde mental.
\end{abstract}

Palavras-chave: adolescência, delinquência juvenil, técnicas psicoterapêuticas.

\section{The reflexive function in adolescents in conflict with the law and school adolescents}

\begin{abstract}
Reflexive Function is identified as an important construct for psychopathological understanding and for clinical practice. The aim of this study was to increase the knowledge about Reflexive Function in adolescents with different trajectories: a group of adolescents in conflict with the law $(\mathrm{G} 1=91, \mathrm{M}$ age $=16.57$ years, $\mathrm{SD}=0.77)$ and a group of teenager students $(\mathrm{G} 2=64, \mathrm{M}$ age $=15,89$ anos, $\mathrm{SD}=0,75)$. Instruments were a Sociodemographic Data Questionnaire, the Questionnaire on Reflexive Function for Young People and the Strenghts and Difficulties Questionnaire. Ninety-one non-clinical adolescents and 95 clinical teenagers took part in the study. Univariate analysis showed significant differences in the Reflexive Function considering the interaction between the G1 and $\mathrm{G} 2$ variables and the clinical and nonclinical categories. Thus, non-clinical G2 presented a greater reflex function $(\mathrm{M}=8.60$, $\mathrm{SD}=0.68, \mathrm{~F}=4.66, \mathrm{p}<0.05)$, followed by clinical $\mathrm{G} 1(\mathrm{M}=8.29, \mathrm{SD}=0.68)$. This characteristic in the clinical group of adolescents in conflict with the law points to hyper-inhalation. It is pointed out the need for evaluation and development of the Reflexive Function as prevention in mental health.
\end{abstract}

Keywords: puberty, juvenile delinquecy, psychotherapeutic techniques.

\section{La función reflexiva en adolescentes en conflicto con la ley y en adolescentes escolares}

\section{Resumen}

A funçión reflexiva ha sido identificado como un constructo importante para la comprensión psicopatológica y para una práctica clínica. Se busca ampliar el conocimiento de la función reflexiva en los adolescentes con diferentes trayectorias de desarrollo, grupo de adolescentes en conflicto con la ley $(\mathrm{G} 1=91, \mathrm{M}$ edad 16,57 anos, $\mathrm{DP}=0,77)$, y grupo de adolescentes escolares $(\mathrm{G} 2=64$, $\mathrm{M}$ de edad 15,89 anos, $\mathrm{DP}=0,75)$. Instrumentos: Ficha de datos sociodemográficos, Cuestionario sobre Función Reflexiva pata Jovens y Cuestionario de Capacidad e Dificuldades. Identificou-se 119 adolescentes no clínicos y 95 adolescentes clínicos. A análise univariada apontou que a función reflexiva demonstrou diferencias significativas considerando una interacción entre una variable G1 y G2 en las categorías clínicas y no clínicas. $(\mathrm{M}=8,60 ; \mathrm{DP}=0,68 ; \mathrm{F}=4,66 ; \mathrm{p}<0,05)$, seguido por $\mathrm{G} 1$ clínico $(\mathrm{M}=8,29, \mathrm{DP}=0,68)$. A presencia de elevada función reflexiva no grupo clínico de adolescentes en conflicto con una ley corrobora una característica de hipermentalización. Aponta-se la necessidade de evaluación y desarrollo de la función reflexiva como prevenición en salud mental. Palavras clave: puberdade, delincuencia juvenil, técnicas psicoterapéuticas. 


\section{Introdução}

A saúde mental tem como base os cuidados parentais recebidos nos primeiros anos de vida (Bowlby, 1973/1998), cuja qualidade das relações de vinculação primordiais funciona como base segura para que a criança explore o ambiente com confiança (Bowlby, 1979/2006) tornando-se modelo de interação futura (Maia, Veríssimo, Ferreira, Monteiro, \& Antunes, 2011). Como resultado dessas experiências de apego, desenvolve-se o modelo interno dinâmico ou modelo representacional das interações com as figuras parentais (Bowlby, 1988). Essas representações possuem uma função regulatória, influenciando como o sujeito percebe a si e aos demais e interferindo na interpretação da realidade (Ramires \& Schneider, 2010). A partir disso, adquire-se a função reflexiva ou mentalização (Fonagy, Gergely, Jurist, \& Target, 2002; Fonagy \& Target, 1997).

Fonagy et al. (2002) apontam que mentalização e função reflexiva são constructos associados a aspectos semelhantes e considerados termos intercambiáveis. Referem-se "à capacidade para compreender e interpretar o comportamento humano em termos de estados mentais subjacentes" (Fonagy \& Bateman, 2003, p. 191) e são fundamentais para a organização de self e na regulação dos afetos (Allen \& Fonagy, 2006; Fonagy \& Bateman, 2003; Sharp, et al., 2009).

A capacidade de mentalizar desenvolve-se em um contexto de apego seguro estabelecido com as figuras primárias (Fonagy \& Allison, 2012). Crianças e adolescentes que experenciaram ao longo do desenvolvimento situações como negligência nos cuidados, maus-tratos e violência ou eventos traumáticos acidentais, podem apresentar sequelas importantes que culminam no estabelecimento de estruturas psíquicas desorganizadas e instáveis na vida adulta: incapacidade reflexiva, difusão de identidade (Ammaniti et al., 2012; Kernberg, Weiner, \& Bardenstein, 2003) e mecanismos de defesa primitivos para lidar com as situações de estresse (Fonagy \& Bateman, 2007).

As dificuldades na função reflexiva podem se apresentar por déficits ou por hipermentalização. Os déficits na capacidade de mentalizar dificultam a regulamentação dos afetos e controle dos impulsos (Rossouw, 2015). Na hipermentalização ocorrem interpretações distorcidas e de maneiras excessivas dos estados mentais dos demais, resultando em uma interpretação equivocada da realidade. Ela é predominante em adolescentes com pensamentos rígidos, com suposições automáticas e injustificadas dos estados mentais de si e dos demais (Sharp et al., 2011; 2013; 2016). Adolescentes com traços borderline caracterizam-se por uma capacidade de mentalizar pobre e vulnerável, assim, utilizam-se da hipermentalização como uma alternativa estratégica (Sharp et al., 2011).

Segundo Fonagy e Target (2004), a adolescência é um período capaz de revelar as falhas da vida primária que até então podiam estar ocultas. A adolescência é um momento de ressignificações identitárias (Jordão, 2008) que auxiliam no amadurecimento (Marcelli \& Braconnier, 1989). As transformações podem acarretar perda nas referências, o que afeta a representação de si mesmo, pois esta ainda se encontra em construção (Biazus, 2011). Nesse sentido, a capacidade de mentalização auxilia na elaboração das transformações e na regulação dos afetos (Ensink et al., 2015; Fonagy et al., 2002).

A adolescência é a faixa etária propícia para o surgimento dos problemas de saúde mental (PSM) (Teodoro, Hess, Saraiva, \& Cardoso, 2014). Esses problemas são comuns e manifestam-se em dificuldades comportamentais, emocionais, sociais e/ou escolares. E, se não forem tratados, podem favorecer prejuízos na vida adulta (Saur \& Loureiro, 2012). Há sintomas internalizantes, que se expressam em relação ao próprio indivíduo e caracterizam-se por comportamentos introspectivos como ansiedade, depressão, isolamento, baixa autoestima, tristeza, timidez e insegurança. E os sintomas externalizantes, que se expressam em relação a outras pessoas e compreendem comportamentos capazes de serem visualizados como, por exemplo, impulsividade, agressividade física e verbal, hiperatividade, delinquência (Achenbach, 1991).

Em relação à etiologia dos PSM, variáveis ambientais e individuais se fazem presentes (Gauy \& Rocha, 2014). Os fatores de risco referem-se a conflitos conjugais (Benetti, 2006; Hess \& Falcke, 2013), baixo nível socioeconômico (Borsa et al., 2011; Sá, Bordin, Martin, \& Paula, 2010), dificuldades físicas, desemprego e baixa escolaridade parental, disciplina inconsistente, negligência, falta de rotina familiar (Gauy \& Rocha, 2014), divórcio dos pais e escola pública em detrimento da particular (Borsa et al., 2011). Especificamente os fatores de risco para os sintomas internalizantes referem-se a situações como violência intrafamiliar grave, punição física aos filhos, embriaguez do pai/padrasto. E, para sintomas externalizantes, acontecimentos como punição física aos filhos, violência física conjugal, desemprego materno e ideação suicida materna (Sá et al., 2010). Ainda, violência física contra criança/adolescente (Cicchetti \& Toth, 2005), dificuldade dos pais no manejo e reconhecimento das próprias emoções (Sá et al., 2010). 
Apesar das condutas de risco serem consideradas frequentes na clínica com adolescentes (Chanen \& McCutcheon, 2013), os dados revelam que quando comparados com a população em geral, adolescentes que cometeram atos infracionais demonstram maior resistência e predominância de aspectos depressivos, comportamentos excêntricos e incomuns, com poucos recursos eficientes para lidar com as demandas da vida diária (Gacono, Vans, \& Viglione, 2008).

Além disso, é predominante nesses sujeitos características de imaturidade na modulação dos afetos (Olin \& Keatinge, 1998) e impossibilidades de conter e situar limites aos excessos pulsionais (Arneklev, Grasmick, Tittle, \& Bursik, Jr., 1993; Rosa Júnior, 2006), gerando sentimentos de raiva e agressão que, muitas vezes, são exercidos como conduta natural de resolução de problemas sendo, portanto, pouco consciente do seu papel nocivo nas relações interpessoais (Gacono \& Meloy, 1994; Olin \& Keatinge, 1998). Observa-se também baixo remorso, culpa ou arrependimento pelo ato realizado (Loving \& Russell, 2000; Olin \& Keatinge, 1998) visto sentimentos deficitários de empatia pelo próximo, o que pode levar à repetição de seus atos e facilitar a reincidência (Martins \& Pillow, 2008).

Apesar da relutância em identificar problemas emocionais e comportamentais, tal avaliação torna-se importante uma vez que eles afetam o desenvolvimento em distintas áreas da vida (Grossi, Moura, Sampaio, \& Silva, 2010). A prevenção que deve se dar em níveis familiar, escolar e individual (Feitosa et al., 2011) tende a favorecer o desenvolvimento da escolaridade, a diminuição do uso e abuso de substâncias psicoativas, a diminuição do ingresso e reincidência na criminalidade e o desenvolvimento de possibilidades de intervenção frente a vulnerabilidades sociais. Dessa forma, este trabalho visa ampliar o conhecimento sobre as características da função reflexiva em adolescentes com diferentes trajetórias de desenvolvimento. Possui o objetivo geral de identificar as características da função reflexiva em adolescentes em conflito com a lei e em adolescentes escolares. Busca, ainda, identificar as variáveis sociodemográficas nos diferentes grupos e avaliar os sintomas emocionais, de conduta, hiperatividade, relacionamento com colegas e comportamento pró-social nos grupos escolares e clínicos correlacionando com a FR.

\section{Método}

Trata-se de um estudo quantitativo, descritivo, transversal (Santos, 1999) e comparativo (Gil, 2008). Os instrumentos utilizados foram: Ficha de
Dados Sociodemográfica, Questionário sobre Função Reflexiva para Jovens (Sharp et al., 2009) e Questionário de Capacidades e Dificuldades (Goodman, 1997; Fleitlich, Cortazar, \& Goodman, 2000).

O Questionário sobre Função Reflexiva para Jovens (Reflective Function Questionnaire for Youths/ RFQY) é um instrumento de autorrelato com 46 itens em escala Likert de seis pontos, que investiga o nível de função reflexiva do sujeito. O escore final aponta que quanto maior o escore, maior a capacidade de função reflexiva (Sharp et al., 2009). A validação junto a norte-americanos, com 146 adolescentes em internação psiquiátrica, verificou que quanto maior a frequência de sintomas de transtorno borderline, menor a capacidade de função reflexiva. Para esse estudo americano a consistência interna do instrumento foi de 0.71 (Ha et al., 2013). Para a utilização do instrumento foi solicitada autorização para a tradução e desenvolvimento da versão em português brasileiro, em processo de validação atualmente. Nesta pesquisa, o RFQY apresentou um $\alpha=0,60$.

O Questionário de Capacidades e Dificuldades (Stregths and Difficulties Questionnaire/ SDQ) é um instrumento norte-americano desenvolvido por Goodman em 1997. Avalia a saúde mental de crianças e adolescentes considerando acontecimentos dos últimos seis meses, a partir de 25 itens em três pontos da escala Likert; desses, 10 itens referem-se às capacidades, 14, às dificuldades e um item é neutro. São divididos em cinco subescalas: sintomas emocionais, problemas de conduta, hiperatividade, problemas de relacionamento com colegas e comportamento pró-social. Ao final, a ocorrência dos sintomas classifica o sujeito entre normal, limítrofe e anormal (Goodman, 1997; Fleitlich, Cortazar, \& Goodman, 2000). Há propriedades psicométricas adequadas para a população brasileira (Fleitlich, Cortazar, \& Goodman, 2000). Para esta pesquisa foi adquirido um $\alpha=0,87$ e considerou-se a classificação limítrofe e anormal como "clínico", enquanto que para a classificação normal utiliza-se a terminologia "não clínico".

A amostra, escolhida por conveniência, refere-se a adolescentes em conflito com a lei (G1) e a adolescentes escolares (G2). Os adolescentes do G1 estavam internados em uma Unidade de Atendimento Socioeducativo devido a ato infracional sentenciado. O G2 se refere a adolescentes matriculados na rede municipal de Ensino Educacional. Em ambos locais foram obtidos a autorização para a coleta de dados dos responsáveis legais e dos adolescentes.

O pressuposto de distribuição normal dos dados foi verificado pelo teste de Kolmogorov-Smirnov. Foi utilizada a estatística descritiva por meio de 
análises de frequência, médias e desvio padrão. Para as comparações foi utilizada estatística paramétrica a partir do método estatístico univariado, em que se descreve a população examinando uma variável por vez, fazendo estimativas das variáveis e desfecho.

Em relação aos aspectos éticos, o projeto foi aprovado no Comitê de Ética da instituição de Ensino (050/2015) vinculada e foi assinado o Termo de Consentimento Livre e Esclarecido (TCLE). O G1 foi autorizado pelo presidente da Fundação de Atendimento Socioeducativo (FASE) e pela diretora da unidade, responsável legal pelos adolescentes; e o G2 recebeu autorização dos pais. Assim como todos foram convidados para a participação voluntária à pesquisa assinando o Termo de Assentimento Livre e Esclarecido (TALE), podendo haver desistência em qualquer momento da coleta. Todos os adolescentes com escore limítrofe ou clínico foram encaminhados para atendimento psicológico.

\section{Resultados}

Participaram deste estudo um total de 214 adolescentes divididos em dois grupos, conforme Tabela 1.

$\mathrm{Na}$ análise univariada, identificou-se que a FR apresentou diferenças significativas considerando a interação entre a variável G1 e G2 nas categorias clínico e não clínico. Desta forma, o G2 não clínico apresentou maior FR $(\mathrm{M}=8,60 ; \mathrm{DP}=0,68 ; \mathrm{F}=4,66$; $\mathrm{p}<0,05)$, seguido pelo $\mathrm{G} 1$ clínico $(\mathrm{M}=8,29 ; \mathrm{DP}=0,68$; Tabela 2). Assim como houve diferença significativa no nível de função reflexiva quanto às demais subescalas do SDQ. Seguem os dados na Tabela 2.

TABELA 1

Variáveis sociodemográficas e clínicas

\begin{tabular}{|c|c|c|}
\hline Variável & $\begin{array}{l}\text { Grupo } 1 \\
\text { Adolescentes em conflito com a lei }\end{array}$ & $\begin{array}{l}\text { Grupo } 2 \\
\text { Adolescentes escolares }\end{array}$ \\
\hline Amostra & 91 & 64 \\
\hline PSM & $\begin{array}{l}\text { Clínico }=38 \\
\text { Não Clínico }=53\end{array}$ & $\begin{array}{l}\text { Clínico }=26 \\
\text { Não Clínico }=38\end{array}$ \\
\hline Idade Média & $16,32(\mathrm{DP}=0,76)$ & $15,72(\mathrm{DP}=0,98)$ \\
\hline Anos de Estudo & Média 8,47 $(\mathrm{DP}=2,26)$ & Média $10,04(\mathrm{DP}=0,85)$ \\
\hline Reprovação & $80(88 \%)$ & $39(31,7 \%)$ \\
\hline Mora com quem & $\begin{array}{l}\text { Mora com pai }=22(24,1 \%) \\
\text { Mora com mãe }=34(37,3 \%) \\
\text { Mora com ambos }=19(20,8 \%) \\
\text { Mora com outros }=35(38,4 \%)\end{array}$ & $\begin{array}{l}\text { Mora com pai }=21(32,8 \%) \\
\text { Mora com mãe }=32(50 \%) \\
\text { Mora com ambos }=17(26,1 \%) \\
\text { Mora com outros }=11(17,1 \%)\end{array}$ \\
\hline Relacionamento dos pais & $\begin{array}{l}\text { Casados }=57(62,6 \%) \\
\text { Separados }=12(13,2 \%) \\
\text { Viúvos }=11(12,1 \%) \\
\text { Solteiros }=11(12,1 \%)\end{array}$ & $\begin{array}{l}\text { Casados }=32(50 \%) \\
\text { Separados }=22(34,3 \%) \\
\text { Viúvos }=2(3,1 \%) \\
\text { Solteiros }=8(12,5 \%)\end{array}$ \\
\hline Escolaridade do pai & $\begin{array}{l}\text { Sem instrução }=9(9,9 \%) \\
\text { Ensino Fundamental = 42 }(46,2 \%) \\
\text { Ensino Médio }=7(7,7 \%) \\
\text { Ensino Técnico }=2(2,2 \%) \\
\text { Não responderam }=31(34,6 \%)\end{array}$ & $\begin{array}{l}\text { Sem instrução = } 1(1,5 \%) \\
\text { Ensino Fundamental = } 19(29,6 \%) \\
\text { Ensino Médio }=20(31,2 \%) \\
\text { Ensino Técnico ou Superior }=18(28,1 \%) \\
\text { Não responderam }=6(9,3 \%)\end{array}$ \\
\hline Escolaridade da mãe & $\begin{array}{l}\text { Sem instrução }=4(4,4 \%) \\
\text { Ensino Fundamental }=49(53,8 \%) \\
\text { Ensino Médio }=15(16,5 \%) \\
\text { Ensino Técnico }=2(2,2 \%) \\
\text { Não responderam }=21(32,8 \%)\end{array}$ & $\begin{array}{l}\text { Sem instrução }=1(1,5 \%) \\
\text { Ensino Fundamental = } 24(37,5 \%) \\
\text { Ensino Médio }=19(29,6 \%) \\
\text { Ensino Técnico ou Superior }=10(15,6 \%) \\
\text { Não responderam }=10(15,6 \%)\end{array}$ \\
\hline
\end{tabular}

Nota: produzido pelas autoras. 
TABELA 2

Análise univariada: médias do nível de FR

\begin{tabular}{|c|c|c|c|c|c|c|}
\hline \multirow{2}{*}{ Problema de Saúde Mental - SDQ } & \multicolumn{2}{|c|}{ G1: em conflito com a lei } & \multicolumn{2}{|c|}{ G2: estudantes } & \multicolumn{2}{|c|}{ Total } \\
\hline & Não-Clínico & Clínico & Não-Clínico & Clínico & Não-Clínico & Clínico \\
\hline Stress Total & $\begin{array}{c}8,26 \\
(\mathrm{DP}=0,65)^{*}\end{array}$ & $\begin{array}{c}8,29 \\
(\mathrm{DP}=0,68)^{*}\end{array}$ & $\begin{array}{c}8,60 \\
(\mathrm{DP}=0,68)^{*}\end{array}$ & $\begin{array}{c}8,19 \\
(\mathrm{DP}=0,79)^{*}\end{array}$ & $\begin{array}{c}8,40 \\
(\mathrm{DP}=0,66)\end{array}$ & $\begin{array}{c}8,29 \\
(\mathrm{DP}=0,63)\end{array}$ \\
\hline Subescala: Sintomas emocionais & $\begin{array}{c}8,33 \\
(\mathrm{DP}=0,62)\end{array}$ & $\begin{array}{c}8,20 \\
(\mathrm{DP}=0,62)\end{array}$ & $\begin{array}{c}8,46 \\
(\mathrm{DP}=0,63)\end{array}$ & $\begin{array}{c}8,16 \\
(\mathrm{DP}=0,85)\end{array}$ & $\begin{array}{c}8,47 \\
(\mathrm{DP}=0,64)^{*}\end{array}$ & $\begin{array}{c}8,18 \\
(\mathrm{DP}=0.68)^{*}\end{array}$ \\
\hline Subescala: Problemas de conduta & $\begin{array}{c}8,39 \\
(\mathrm{DP}=0,63)\end{array}$ & $\begin{array}{c}8,23 \\
(\mathrm{DP}=0,61)\end{array}$ & $\begin{array}{c}8,63 \\
(\mathrm{DP}=0,64)\end{array}$ & $\begin{array}{c}8,22 \\
(\mathrm{DP}=0,83)\end{array}$ & $\begin{array}{c}8,53 \\
(\mathrm{DP}=0,65)^{*}\end{array}$ & $\begin{array}{c}8,23 \\
(\mathrm{DP}=0,63)^{*}\end{array}$ \\
\hline $\begin{array}{l}\text { Subescala: Hiperatividade e problemas de } \\
\text { concentração }\end{array}$ & $\begin{array}{c}8,31 \\
(\mathrm{DP}=0,65)^{*}\end{array}$ & $\begin{array}{c}8,26 \\
(\mathrm{DP}=0,54)^{*}\end{array}$ & $\begin{array}{c}8,59 \\
(\mathrm{DP}=0,71)^{*}\end{array}$ & $\begin{array}{c}8,55 \\
(\mathrm{DP}=0,55)^{*}\end{array}$ & $\begin{array}{c}8,43 \\
(\mathrm{DP}=0,68)\end{array}$ & $\begin{array}{c}8,37 \\
(\mathrm{DP}=0,55)\end{array}$ \\
\hline $\begin{array}{l}\text { Subescala: Problema de relacionamento } \\
\text { com colegas }\end{array}$ & $\begin{array}{c}8,33 \\
(\mathrm{DP}=0,62)\end{array}$ & $\begin{array}{c}8,20 \\
(\mathrm{DP}=0,62)\end{array}$ & $\begin{array}{c}8,58 \\
(\mathrm{DP}=0,72)^{*}\end{array}$ & $\begin{array}{c}8,59 \\
(\mathrm{DP}=0,55)^{*}\end{array}$ & $\begin{array}{c}8,44 \\
(\mathrm{DP}=0,68)\end{array}$ & $\begin{array}{c}8,38 \\
(\mathrm{DP}=0,62)\end{array}$ \\
\hline Subescala: Comportamento Pró-Social & $\begin{array}{c}8,34 \\
(\mathrm{DP}=0,62)\end{array}$ & $\begin{array}{c}8,20 \\
(\mathrm{DP}=0,61)\end{array}$ & $\begin{array}{c}8,47 \\
(\mathrm{DP}=0,62)\end{array}$ & $\begin{array}{c}8,15 \\
(\mathrm{DP}=0,85)\end{array}$ & $\begin{array}{c}8,51 \\
(\mathrm{DP}=0,65)\end{array}$ & $\begin{array}{c}8,22 \\
(\mathrm{DP}=0,63)\end{array}$ \\
\hline Sintomas internalizantes & $\begin{array}{c}8,39 \\
(\mathrm{DP}=0,61)\end{array}$ & $\begin{array}{c}8,21 \\
(\mathrm{DP}=0,63)\end{array}$ & $\begin{array}{c}8,61 \\
(\mathrm{DP}=0,64)\end{array}$ & $\begin{array}{c}8,20 \\
(\mathrm{DP}=0,84)\end{array}$ & $\begin{array}{c}8,41 \\
(\mathrm{DP}=0,68)\end{array}$ & $\begin{array}{c}8,34 \\
(\mathrm{DP}=0,52)\end{array}$ \\
\hline Sintomas externalizantes & $\begin{array}{c}8,20 \\
(\mathrm{DP}=0,61)\end{array}$ & $\begin{array}{c}8,33 \\
(\mathrm{DP}=0,62)^{*}\end{array}$ & $\begin{array}{c}8,56 \\
(\mathrm{DP}=0,71)\end{array}$ & $\begin{array}{c}8,52 \\
(\mathrm{DP}=0,55)\end{array}$ & $\begin{array}{c}8,43 \\
(\mathrm{DP}=0,68)\end{array}$ & $\begin{array}{c}8,35 \\
(\mathrm{DP}=0,62)\end{array}$ \\
\hline
\end{tabular}

$* \mathrm{p}<0,05$.

\section{Discussão}

A adolescência é um período de transformações em diferentes áreas que precisam ser elaboradas (Aberastury \& Knobel, 1992). Entretanto, sobrecarregas da capacidade simbólica em lidar com os sentimentos e pensamentos podem desencadear sofrimento psíquico, sintomatologia somática, transtornos mentais e atuações físicas como condutas agressivas (Biazus \& Ramires, 2012). Na amostra em questão, observa-se um alto nível de sintomatologia clínica, o que, nessa faixa etária, é indicativa de prognóstico clínico (Crawford et al., 2008). Nesse sentido, diagnósticos de doença mental na vida adulta demonstram uma estabilidade de problemas emocionais e de conduta desde a adolescência (Castañeda \& Gómez-Becerra, 2012). Assim como os sintomas e as deficiências não diminuem a prevalência com a idade, quando comparados até 20 anos mais tarde (Johnson et al., 2000).

As variáveis sociodemográficas, no geral, não foram determinantes para o desenvolvimento da função reflexiva no grupo em estudo. Porém, segundo alguns autores, (Crawford et al., 2008; Paris, 2013) podem estar associadas aos PSM. Morar e conviver com o pai e mãe, por sua vez, são apontados como um fator protetivo diante de vulnerabilidades (Paiva \& Ronzani, 2009). Destaca-se no G1 que um menor número de sujeitos relatou morar com tais figuras de vinculação primária (pai e mãe). Tal característica pode associar- se à dificuldade na efetivação dos cuidados parentais e ao comprometimento da qualidade do vínculo familiar, amparo, proteção e limites (Rovinski \& Cruz, 2009; Zappe \& Dias, 2012).

Da mesma forma, evidencia-se menor escolaridade dos pais no G1, indicando que o sucesso escolar, na maioria das vezes, é uma meta pouco relevante, não sendo desejado e esperado em relação aos filhos, portanto pouco valorizado e incentivado pelo meio (Davoglio \& Gauer, 2011). Demonstram-se, ainda, índices de desemprego mais elevados associados à baixa escolaridade (Cabral \& Sousa, 2014) e que a baixa escolaridade paterna e materna pode ser considerada um fator de risco para o desenvolvimento de crianças e adolescentes, sendo ampliada a ocorrência de PSM (Davoglio \& Gauer, 2011).

Em relação às idades, percebe-se que há semelhanças entre o G1 e o G2; porém, há menor média de anos de estudo e maior número de reprovações no G1. Tal grupo está cerca de três anos abaixo da escolaridade esperada para a idade, o que pode corroborar com o a desistência e desmotivação, o que para Pereira e Sudbrack (2009) influencia o adolescente a procurar por outras perspectivas de futuro. A instituição escolar, apesar de considerada um local de proteção ao desenvolvimento com relevante papel social (Gallo \& Williams, 2008), em determinados contextos, demonstra uma indisponibilidade de aceitação ou dificuldades em lidar com adolescentes que apresentem mau desempenho e fracasso escolar, 
acarretando em abandono (Legnani, 2009). Dados da literatura indicam que a faixa etária entre 15 e 17 anos é considerada a mais exposta a comportamentos de risco, atitudes impulsivas, aproximação com grupos de amigos, exposição ao uso de substâncias psicoativas e violência, o que pode influenciar o envolvimento em atos infracionais (Carvalho \& Gomide, 2005).

Ainda, tem-se que, quando os sujeitos se expõem ou vivenciam situações traumáticas em seus desenvolvimentos ou até mesmo em momentos atuais, sofrem um déficit na capacidade da função reflexiva (Bateman \& Fonagy, 2006; Fonagy \& Target, 1997). A FR é definida como capacidade do sujeito em reconhecer pensamentos, sentimentos, crenças e desejos próprios e dar significado ao comportamento do outro, permitindo prever e interpretar intenções e sentimentos (Bateman \& Fonagy, 2010).

Nos adolescentes em questão, observa-se que os adolescentes escolares com escore não clínico apresentaram maior nível de função reflexiva, dessa forma infere-se que possuíram trajetórias de vida mais estáveis, sem ocorrência de traumas severos e negligências no período de seus desenvolvimentos. $\mathrm{O}$ que proporcionou o estabelecimento do apego seguro e o desenvolvimento da função reflexiva (Fonagy \& Allison, 2012). Entretanto, chama a atenção que os adolescentes em conflito com a lei com escore não clínico, também apresentaram nível de função reflexiva mais alta, o que indica características de hipermentalização (Quiroga \& Cryan, 2013).

A hipermentalização é entendida como a ocorrência de interpretações excessivas dos estados mentais dos demais, sendo que o sujeito distorce e interpreta a realidade de maneira equivocada. É comum o predomínio de suposições irreflexivas, rígidas e automáticas sustentadas com certezas injustificadas dos estados mentais internos e dos demais. Além disso, centra-se em demasia nos aspectos externos deles mesmos e dos outros (Sharp et al., 2011; 2013; 2016).

Em relação a adolescentes em conflito com a lei, dados revelam que quando comparados com a população em geral, os mesmos possuem maior resistência e predominância de aspectos depressivos, comportamentos excêntricos e incomuns, com poucos recursos eficientes para lidar com as demandas da vida diária (Gacono, Vans, \& Viglione, 2008). Além disso, é predominante nesses sujeitos características de imaturidade na modulação dos afetos (Olin \& Keatinge, 1998) e impossibilidades de conter e situar limites aos excessos pulsionais (Arneklev, Grasmick, Tittle, \& Bursik, Jr., 1993; Rosa Júnior, 2006), gerando sentimentos de raiva e agressão. Tais características são exercidas como conduta natural de resolução de problemas sendo, portanto, pouco conscientes do seu papel nocivo nas relações interpessoais (Gacono \& Meloy, 1994; Olin \& Keatinge, 1998). De tal forma supõe-se que não tenham desenvolvido adequada regulação emocional e, consequentemente, a FR.

Destaca-se que, neste estudo, o ato infracional em si não foi exclusivamente determinante para o nível menor de função reflexiva, mas sim a presença de sintomas emocionais e problemas de conduta, ou seja, existência de sintomatologia clínica. Neste sentido, observa-se que um sujeito em sofrimento emocional possui limitada capacidade de refletir sobre si e os demais e compreender o que realmente se passa consigo e com o ambiente, dessa forma, reagindo com problemas de conduta, tais como: impaciência, desobediência às regras, imposição, entre outros.

Ainda é possível dizer que devido às situações de maior vulnerabilidade enfrentadas durante seus desenvolvimentos, alguns, utilizam-se de maneira excessiva a mentalização como uma alternativa para "sobrevivência" (Sharp et al., 2011). Pesquisas anteriores apontam o predomínio de hipermentalização em 259 participantes, com idade média de 15 anos, e sua associação com vínculo inseguro e desregulação emocional, características indicativas de traços de personalidade borderline (Sharp et al., 2016). Em outra amostra com 111 internos de uma clínica psiquiátrica, com idade média de 15,5 anos, evidenciou-se que os adolescentes, também com traços borderline, caracterizavam-se por mentalização pobre e utilizavam-se da hipermentalização como uma alternativa estratégica. Tal aspecto acontece independentemente da idade, gênero e existência de sintomas internalizantes ou externalizantes (Sharp et al., 2011). Os achados causam, então, inquietações, visto os desvios no desenvolvimento saudável dos sujeitos, reforçando a importância de atuar de modo preventivo e curativo nesta fase do desenvolvimento, já que pesquisadores (Liotti \& Gilbert, 2010; Sharp et al., 2013) declaram que adolescentes apresentam potencial elevado de mudança e ampliação da capacidade mentalizadora.

Concomitantemente a isso, o que realmente associase ao ato infracional é a ocorrência de problemas de relacionamento com os colegas, problemas de atenção e hiperatividade, já que quando há esses sintomas, o nível de FR apresenta-se baixo. Dessa forma, verificase que adolescentes que demonstram maior sentimento de solidão, baixo ou nenhum apoio do semelhante ou/e que conta com poucos recursos protetivos de ordem pública, assim como comportamento inquieto, agitado e com concentração prejudicada, podem estar expostos a maior risco para o envolvimento em atos infracionais. 
Denota-se assim, que a FR pode ser considerada um fator de proteção e que seu desenvolvimento se torna fundamental para as transformações vivenciadas na adolescência e para auxiliar na elaboração das perdas características da adolescência, mantendo o indivíduo seguro e estável (Fonagy et al., 2002; Taubner, White, Zimmermann, Fonagy, \& Nolte, 2013).

Algumas limitações devem ser observadas. Os resultados foram baseados em adolescentes do sexo masculino. $\mathrm{O}$ instrumento baseia-se em autorrelato, podendo os achados serem mais abrangentes a partir de dados de diferentes informantes e através de múltiplos métodos. Em relação ao instrumento RFQY, ainda não validado no Brasil, há consistência interna fraca que precisa ser trabalhada para se tornar uma medida mais efetiva. Aponta-se a necessidade de investir em mais pesquisa abrangendo adolescentes em diferentes contextos e diferentes características pessoais para consolidação dos resultados. Também, a necessidade de desenvolvimento de medidas psicométricas que auxiliem a avaliação da FR em adolescentes, já que déficits ou hipermentalização na FR tem sido apontados como centrais em transtornos de personalidade (Kernberg et al., 2003), em adolescentes depressivos (Fischer-Kern et al., 2008), psicóticos (Brent, 2015; Sharp, 2006), vítimas de negligência e violência (Bateman \& Fonagy, 2006), entre outros.

\section{Considerações finais}

Através dos resultados demonstrou-se a importância de avaliar adolescentes aprofundando a compreensão dos aspectos intrapsíquicos, identificando sintomas e características da FR, levantando possíveis fatores de risco que podem se relacionar ao desenvolvimento de psicopatologia. Nesse caso, aponta-se a importância de intervenções clínicas voltadas para a avaliação e desenvolvimento da FR, ampliando possibilidades de prevenção e de mudança terapêutica. Particularmente frente ao tema do adolescente em conflito com a lei, aponta-se a complexidade do assunto e a importância da investigação contínua auxiliando nas reflexões frente a intervenções mais apropriadas sobre o funcionamento do indivíduo.

\section{Referências}

Aberastury, A., \& Knobel, M. (1992). Adolescência normal: um enfoque psicanalítico. Porto Alegre: Artes Médicas. (DOI INEXISTENTE)

Achenbach, T. M. (1991). Manual for the Child Behavior Checklist/4-18 e 1991 Profile. Burlington, VT: University of Vermont Department of Psychiatry. (DOI INEXISTENTE)

Allen, J. P., \& P. Fonagy (2006). The Handbook of Mentalization Based Treatment. Chichester: John Wiley \& Sons. doi:10.1002/9780470712986

Ammaniti, F., Massimo, A., Clarkin, A., Clarkin, J. F., Nicolais, G., \& Kernberg, O. F. (2012). Assessment of adolescent personality disorders through the interview of personality organization processes in adolescence (IPOP-A): Clinical and Theoretical Implications. Adolescent Psychiatry, 2, 36-45. doi:10.2174/2210676611202010036

Arneklev B. J., Grasmick H. G., Tittle C. R., \& Bursik Jr. R. J. (1993). Low Self-Control and Imprudent Behavior. Journal of Quantitative Criminology, 9(3), 225-247. doi:10.1080/01639621003800505

Bateman A. \& Fonagy P. (2010). Mentalization based treatment for borderline personality disorder: special article. World Psychiatry, 9, 11-15. doi:10.1002/j.2051-5545.2010.tb00255.x

Benetti, S. P. C. (2006). Conflito conjugal: Impacto no desenvolvimento psicológico da criança e do adolescente Psicologia: Reflexão e Crítica, 19(2), 261-268. doi:10.1590/S0102-79722006000200012

Biazus, C. B. \& Ramires, V. R. R. (2012). Depressão na adolescência: uma problemática dos vínculos. Maringá: Psicologia em Estudo, 17(1), 83-91. doi:10.1590/S1413-73722012000100010

Biazus, C. B. (2011). A psicoterapia baseada na mentalização para o tratamento da depressão na adolescência (Dissertação de mestrado). Universidade do Vale do Rio dos Sinos, Programa de Pós-Graduação em Psicologia Clínica,, São Leopoldo, RS. (DOI INEXISTENTE)

Borsa, J. C., Souza, D. S. de, \& Bandeira, D. R. (2011). Prevalência dos problemas de comportamento em uma amostra de crianças do Rio Grande do Sul. Psicologia: Teoria e Prática, 13(2), 15-29. (DOI INEXISTENTE)

Bowlby, J. (1973/1998). Apego e perda: Separação: angústia e raiva. (2. Ed.). São Paulo, SP: Martins Fontes. (DOI INEXISTENTE)

Bowlby, J. (1979/2006). Formação e rompimento dos laços afetivos. São Paulo, SP: Martins Fontes. (DOI INEXISTENTE)

Bowlby, J. (1988). Uma base segura: aplicações clínicas da teoria do apego. Porto Alegre, RS: Artmed. (DOI INEXISTENTE) 
Brent, B. K. (2015). A mentalization-based approach to the development of the therapeutic aliance in the treatment of schizophenia. Journal of Clinical Psychology, 71(2), 1-11. doi:10.1002/jclp.22150

Cabral S. H. \& Sousa S. M. G. (2014). O histórico processo de exclusão/inclusão dos adolescentes autores de ato infracional no Brasil. Psicologia em Revista, 10(15), 71-90. (DOI INEXISTENTE)

Carvalho, M. C. N. \& Gomide, P.I.C. (2005). Práticas educativas parentais em famílias de adolescentes em conflito com a lei. Estudos de Psicologia, 22(3), 263-275. doi:10.1590/S0103-166X2005000300005

Castañeda, D. R. \& Gómez-Becerra, I. (2012). Patrones de personalidad disfuncionales em niños y adolescentes: uns revisón funcional - contextual. Suma Psicológica, 19(2), 131-149. doi:10.14349/sumapsi2012.1125

Chanen, A. M. \& McCutcheon, L. (2013). Prevention and early intervention for borderline personality disorder: Current status and recent evidence. The British Journal of Psychiatry, 54, 24-9. doi:10.1192/bjp.bp.112.119180

Cicchetti, D. \& Toth, S. L. (2005). Child maltreatment. Annual Review of Clinical Psychology, 1, 409-438. doi:10.1146/ annurev.clinpsy.1.102803.144029

Crawford, T. N., Cohen P., First, M. B., Skodol, A. E., Johnson, J. G., \& Kasen S. (2008). Comorbid Axis I and Axis II disorders in early adolescence. Archives of General Psychiatry, 65, 641-648. doi:10.1001/archpsyc.65.6.641

Davoglio T. R. \& Gauer, G. J. C. (2011). Adolescentes em conflito com a lei: aspectos sociodemográficos de uma amostra em medida socioeducativa com privação de liberdade. Contextos Clínicos, 4(1), 42-52. doi:10.4013/ ctc. 2011.41 .05

Ensink, K., Fonagy, P., Normandin, L., Berthelot, N., \& Duval, J. (2015). O papel protetor da mentalização de experiências traumáticas: implicações quando da entrada na parentalidade. Estilos clínicos, 20(1), 76-91. doi:10.11606/issn.19811624.v20i1p76-91

Feitosa, H. N., Ricou, M., Rego, M., \& Nunes, R. (2011). A saúde mental das crianças e dos adolescentes: considerações epidemiológicas, assistenciais e bioéticas. Revista bioética 19(1), 259-275. (DOI INEXISTENTE)

Fischer-Kern, M., Tmej, A., Kapusta, N. D., Naderer, A., Leithner-Dziubas, K., Loffer-Staska, H., \& Springer-Kremser, M. (2008). The capacity for mentalization in depressive patients: A pilot study. Psychosomatic Medicine and Psychotherapy, 54(4), 368-380. doi:19049686

Fleitlich, B. W., Cortazar, P. G., \& Goodman, R. (2000). Questionário de Capacidades e Dificuldades (SDQ). Infanto, 8(1), 44-50. (DOI INEXISTENTE)

Fonagy, P. \& Allison, E. (2012). What is mentalization? The concept and its foundation in developmental research. In Midgley, N., \& Vrouva, I, Minding the Child (pp. 11-34). London: Routledge. (DOI INEXISTENTE)

Fonagy, P. \& Bateman, A. W. (2003). The development of na attachment-based treatment program for borderline personality disorder. Bulletin of the Menninger Clinic, 67(3), 187-211. doi:10.1521/bumc.67.3.187.23439

Fonagy, P. \& Bateman, A. W. (2007). Mentalizing and borderline personality disorder. Journal of Mental Health, 16(1), 83-101. doi:10.1080/09638230601182045

Fonagy, P. \& Target, M. (1997). Attachment and reflective function: Their role in self-organization. Development and Psychopathology, 9, 679-700. doi: 10.1017/S0954579497001399

Fonagy, P. \& Target, M. (2004). Questões desenvolvimentais na adolescência normal e colapso na adolescência. In Graña, R. B., \& Piva, A. B. S. (Orgs.), A atualidade da psicanálise de adolescentes - formas do mal-estar na juventude contemporânea (pp. 91-106). São Paulo, SP: Casa do Psicólogo. (DOI INEXISTENTE)

Fonagy, P., Gergely, G., Jurist, E. L., \& Schafer, W. D. (2002). Affect regulation, mentalization, and the development of the self. Nova Yorque, NY: Other (DOI INEXISTENTE)

Gacono, C. B. \& Meloy, J. R. (1994). The Rorschach Assessment of Aggressive and Psychopathic Personalities. Hillsdale, NJ: Lawrence Erlbaum Associates (DOI INEXISTENTE)

Gacono, C. B., Evans, F. B., \& Viglione, D. J. (2008). Essential issues in the forensic use of the Rorschach. In C. B. Gacono, F. B. Evans, N. Kaser-Boyd, \& L. A. Gacono (Eds.), Handbook of forensic Rorschach psychology (pp. 3-20). Mahwah, NJ: Lawrence. (DOI INEXISTENTE)

Gallo, A. E.; Williams, L. C. A. (2008). A escola como fator de proteção à conduta infracional de adolescentes. Cadernos de Pesquisa, 38(133), 41-59. doi:10.1590/S0100-15742008000100003

Gauy, F. V. \& Rocha, M. M. (2014) Manifestação clínica, modelos de classificação e fatores de risco/proteção para psicopatologias na infância e adolescência. Temas psicol. [online], 22(4), 783-793. doi:10.9788/TP2014.4-09

Gil, A. C. (2008). Métodos e técnicas de pesquisa social. (6. ed.) São Paulo, SP: Atlas. (DOI INEXISTENTE)

Goodman, R. (1997). The Strenghts and Difficulties Questionnaire: A research note. Journal of Child Psychology and Psychiatry, 38, 581-586. doi:10.1111/j.1469-7610.1997.tb01545.x

Grossi, R., Moura, C. B., Sampaio, A. C. P., \& Silva, L. C. (2010). Questionário de Situações Domésticas: Comparação entre pré-escolares clínicos e não-clínicos. Psicologia: Teoria e Pesquisa, 26(1), 57-66. doi:10.1590/S010237722010000100008

Ha, C., Sharp, C., Ensink, K., Fonagy, P., \& Cirino, P. P. (2013). The measurement of reflective function in adolescents with and without borderline traits. Journal of Adolescence, 36, 1215-1223. doi:10.1111/j.2044-8341.2010. 02006.x

Hess, A. R. B. \& Falcke, D. (2013). Sintomas internalizantes na adolescência e as relações familiares: uma revisão sistemática da literatura. Psico-USF, 18(2), 263-276. doi:10.1590/S1413-82712013000200010 
Johnson, J. G., Cohen, P., Kasen, S., Skodol, A. E., Hamagami, F., \& Brook, J. S. (2000). Age-related change in personality disorder trait levels between early adolescence and adulthood: A communitybased longitudinal investigation. Acta Psychiatrica Scandinavica, 102, 265-275. doi:10.1034/j.1600-0447.2000.102004265.x

Jordão, A. B. (2008). Vínculos familiares na adolescência: Nuances e vicissitudes na clínica psicanalítica com adolescentes. Aletheia, 21(1), 157-172. (DOI INEXISTENTE)

Kernberg, P. F., Weiner, A. S., \& Bardenstein, K. K. (2003). Transtorno de Personalidade em crianças e adolescentes: uma visão geral. Porto Alegre, RS: Artmed. (DOI INEXISTENTE)

Legnani, V. N. (2009) Preconceito e segregação na escola em relação aos usuários de drogas ilícitas. In Caderno de Resumos do XIII Colóquio Internacional de Psicossociologia e Sociologia Clínica, 261-262). (DOI INEXISTENTE)

Liotti, G. \& Gilbert, P. (2010). Mentalizing, motivation, and social mentalities: Theoretical considerations and implications for psychotherapy. Psychology and Psychotherapy: Theory, 84(1), 9-25. doi:10.1348/147608310X520094

Loving, J. \& Russell, W. (2000). Selected Rorschach variables of psychopathic juvenile offenders. Journal of Personality Assessment, 75, 126-142. doi:10.1207/S15327752JPA7501_9

Maia, J., Veríssimo, M., Ferreira, B., Monteiro, L., \& Antunes, M. (2011). Representação de vinculação na infância: competência verbal, estabilidade e mudança. Análise Psicológica, 3(29), 403-424. (DOI INEXISTENTE)

Marcelli, D. \& Braconnier, A. (1989). Manual de psicopatologia do adolescente. Porto Alegre, RS: Artes Médicas. (DOI INEXISTENTE)

Martins, M. \& Pillow, S. (2008). A relação entre a iniciação do uso de drogas e o primeiro ato infracional entre os adolescentes em conflito com a lei. Cadernos de Saúde Pública. 24(5), 1112-1120. doi:10.1590/S0102-311X 2008000500018

Olin, J. \& Keatinge, C. (1998). Rapid psychological assessment. New York, NY: John Wiley. (DOI INEXISTENTE)

Paris, J. (2013). Personality disorders begin in adolescence. Journal of the Canadian Academy of Child and Adolescent Psychiatry, 22(3), 195-196. (DOI INEXISTENTE)

Pereira, S. E. \& Sudbrack, M.F. (2009). A formação dos grupos na adolescência: A escola que exclui. In Caderno de Resumos do XIII Colóquio Internacional de Psicossociologia e Sociologia Clínica (pp. 259-260). (DOI INEXISTENTE)

Quiroga, S. \& Cryan, G. (2012). Análisis de proceso em grupos de terapia focalizada para adolescentes violentos: Función reflexiva - RF y modelo de ciclo terapêutico- TCM. Anuario de Investigaciones, Facultad de Psicología UBA, Secretaría de Investigaciones, 19. (DOI INEXISTENTE)

Ramires, V. R. R. \& Schneider, M. S. (2010). Revisando alguns conceitos da teoria do apego: comportamento versus representação? Psicologia: Teoria e Pesquisa, 26(1), 25-33. doi:10.1590/S0102-37722010000100004

Rosa Júnior N. C. D. (2006). Adolescência e violência: direção do tratamento psicanalítico com adolescentes em conflito com a lei. (Dissertação de mestrado) Faculdade de Psicologia, Universidade Federal do Rio Grande do Sul, Porto Alegre. (DOI INEXISTENTE)

Rossouw, T. I. (2015). The use of mentalization-based treatment for adolescents (TBM-A) witha Young woman with mixed personality disorder and tendencies to self-harm. Journal of Clinical Psychology, 71(2), 178-187. doi:10.1002/ jclp. 22153

Rovinski, S. M. R. \& Cruz, R. M. (Orgs.) (2009). Psicologia jurídica: perspectivas teóricas e processos de intervenção. São Paulo, SP: Vetor. (DOI INEXISTENTE)

Paiva, F. S. \& Ronzani, T.M. (2009). Estilos parentais e consumo de drogas entre adolescentes: revisão sistêmica. Psicologia em estudo, 14(1), 177-183. (DOI INEXISTENTE)

Sá, D. G. F. de, Bordin, I. A. S., Martin, D., \& Paula, C. S. de (2010). Fatores de risco para problemas de saúde mental na infância/adolescência. Psicologia: Teoria e Pesquisa, 26(4), 643-652. doi:10.1590/S0102-37722010000400008

Santos, A. R. dos (1999). Metodologia científica: A construção do conhecimento. Rio de Janeiro, RJ: DP \& A. (DOI INEXISTENTE)

Saur, A. M. \& Loureiro, S. R. (2012). Qualidades psicométricas do Questionário de Capacidades e Dificuldades: Revisão de literatura. Estudos de Psicologia, 29(4), 619-629. doi:10.1590/S0103-166X2012000400016

Sharp et al. (2011). Theory of mind and emotion regulation difficulties in adolescents with borderline traits. Journal of the American Academy of Child \&Adolescent Psychiatry, 50(6), 563-573. doi:10.1016/j.jaac.2011.01.017

Sharp et al. (2013). Hypermentalizing in adolescent inpatients: Treatment effects and association with borderline traits. Journal of Personality Disorders 27(1), 3-18. doi:10.1521/pedi.2013.27.1.3

Sharp et al. (2016). First empirical evaluation of the link between attachment, social cognition and borderline features in adolescents. Comprehensive Psychiatry 64, 4-11. doi:10.1016/j.comppsych.2015.07.008

Sharp, C. (2006). Mentalizing problems in childhood disorders. In J. Allen \&, P. Fonagy (Eds.), Handbook of mentalizationbased treatment (pp. 101-121). Chichester: John Wiley \& Sons. doi:10.1002/9780470712986.ch4

Sharp, C., Williams, L., Ha, C., Baumgardner, J., Michonski, J., Seals, R., Patel, A. B., Bleiberg, E., \& Fonagy, P. (2009). The development of a mentalization-based outcomes and research protocol for an adolescent in-patient unit. Bulletin of the Menninger Clinic, 73, 311-338. doi:10.1521/bumc.2009.73.4.311

Taubner, S., White, L.O., Zimmermann, J., Fonagy, P., Nolte, T. (2013) Attachment-related mentalization moderates the relationship between psychopathic traits and proactive aggression in adolescence. Journal Abnorm Child Psychol. 41(6):929-38. doi: 10.1007/s10802-013-9736-x 
Teodoro, M. L. M., Hess, A. R. B., Saraiva, L. A., \& Cardoso, B. M. (2014). Problemas emocionais e de comportamento e clima familiar em adolescente e seus pais. Psico, 45(2), 168-175. doi:10.15448/1980-8623.2014.2.13172

Zappe, J. G. \& Dias A. C. G. (2012). Violência e fragilidades nas relações familiares: refletindo sobre a situação de adolescentes em conflito com a lei. Estudos de Psicologia, 17(3), 389-395. (DOI INEXISTENTE)

Dados das autoras:

Taís Cristina Favaretto - Mestre, Universidade do Vale do Rio dos Sinos.

Luciane Maria Both - Doutoranda, Universidade Federal do Rio Grande do Sul.

Sílvia Pereira da Cruz Benetti - Doutora, Universidade do Vale do Rio dos Sinos.

Endereço para correspondência:

Taís Cristina Favaretto

Rua Independência, 99a, apto 401 - Centro

Passo Fundo, RS, Brasil.

E-mail: taisfavaretto@gmail.com

Recebido em: 21/08/2017.

Aceito em: 27/08/2018.

Publicado em: 09/05/2019. 